\title{
La comunicación de la innovación de las agencias de medios. Big data en las webs propias
}

institucional.us.es/ambitos/

Natalia Papí-Gálvez

Universidad de Alicante

natalia.p@ua.es

\section{Raquel García-Bonal}

Universidad de Alicante

English Version: The broadcast of media agencies innovations. Big data in corporate intranets

\section{Resumen}

Las agencias de medios constituyen uno de los sectores publicitarios en el que la aplicación de big data proporciona soluciones innovadoras que debieran ser comunicadas a través, entre otros canales, de sus webs corporativas. Con el

propósito de facilitar tal comunicación, las webs deben estar preparadas para ser consultadas por varios dispositivos. Así, este estudio pretende averiguar su uso para comunicar innovaciones relacionadas con big data, cuidando el responsive design, de las 20 primeras agencias de medios españolas, y reflexiona sobre las debilidades, amenazas, fortalezas y oportunidades de tal innovación.

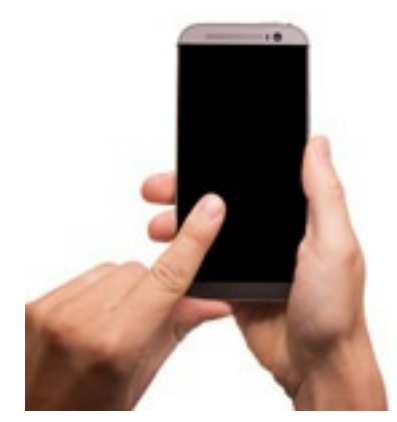

\section{Palabras clave}

Web propia, investigación de medios publicitarios, Big Data, agencias de medios, comunicación de la innovación.

Media agencies are an advertising sector in which proper application of big data provides innovative solutions which should be communicated through, among other channels, of their corporate web sites. With the purpose of

facilitate such communication, those web sites must be ready to be checked with different devices. Thus, this study aims to find its use for communicating innovations related to big data, tending the responsive design, of the first 20 spanish media agencies, and reflects on the weaknesses, threats, strengths and opportunities of this innovation.

Keywords Owned Web, media research, Big Data, media agency, communication of innovation.

\section{INTRODUCCIÓN}

\subsection{Innovación y su comunicación a través de las webs corporativas}

La Unión Europea presenta la innovación como uno de los ejes vertebradores de la Estrategia

Europa 2020 (Comisión Europea, s. f.) para el crecimiento sostenible, social y económico de sus países (Comisión Europea, 2014). Se utiliza el término innovación en un sentido amplio, es decir, como capacidad para la creación y la generación de nuevas ideas, respaldada por el potencial que ofrece la preparación de los ciudadanos y la fuerza de la industria creativa. Europa apuesta por el desarrollo de esta capacidad para la generación de puestos de trabajo y considera fundamental centrarse no sólo en las grandes empresas sino también en las PYMES para conseguirlo (Comisión Europea, 2010). 
España desde el año 2008 demandaba una intervención de carácter urgente. De hecho, el último informe de Cotec (2015) sobre Tecnología e Innovación muestra una drástica reducción hasta 2013 del número de empresas innovadoras y la necesidad de más fondos públicos para su fomento. Dado el conjunto de estándares y medidas puestos en marcha, para 2014 y 2015 se esperan cifras mejores.

La innovación de cualquier tipo es vital para las empresas no sólo porque puedan conseguir ventajas competitivas, como se ha subrayado en numerosas ocasiones, sino sobre todo para activar cambios acompasados con la velocidad de las transformaciones que se producen en la actualidad. Tal es así que la innovación, según Rita G. McGrath (2013), tendría que ser sistemática, en tanto que se trata del camino para obtener lo que la autora llama ventaja competitiva transitoria. En definitiva, se trataría de incorporar las acciones de I+D+i como una operación más, con el propósito de seguir siendo competitivos (Cotec, 2015).

Para el caso concreto de las agencias de medios, el mundo digital ofrece retos cuya asunción puede estar produciendo transformaciones en sus propias funciones. Así, tales organizaciones se convierten en "...centros de aprendizaje acerca de Internet” (Pérez-Latre; Sánchez Tabernero, 2015) como efecto de los cambios que en su propia materia prima se están produciendo (Papí-Gálvez, 2015). La innovación en las mismas es imperativa. Sus servicios se amplían para poder satisfacer las demandas del nuevo mercado, adaptarse a las nuevas reglas de comunicación y aprovechar las oportunidades de negocio que todo ello ofrece.

El proceso de innovación debe concluir en su comunicación, como viene recogiendo Cotec (2004) desde hace más de dos décadas. Es preciso dar a conocer la contribución de la innovación a la sociedad pues, entre otras aplicaciones, es posible construir una excelente reputación basada justamente en los activos de innovación de la empresa (Corporate Excellence, 2015).

A este respecto, la comunicación de la innovación entraña varias fases: desde la puesta en marcha de la misma y la difusión por parte de la empresa, hasta la divulgación de la innovación a través de los medios de comunicación y el conocimiento de las acciones realizadas por parte de la población. Este proceso puede y debe contar con el esquema POES de medios (Paid, Owned, Earned, Shared) utilizado en las estrategias de comunicación actuales.

Las webs corporativas son, por excelencia, el medio propio online de una firma. Presentan (y representan) a la compañía en el mundo digital y es el punto de partida de otros medios que además de propios son sociales o compartidos (por ejemplo, blogs o perfiles en redes sociales). Todos ellos pueden contribuir no sólo a la comunicación externa de la innovación sino también al impulso de la cultura innovadora en su conjunto (Vanhaverbeke; Chesbrough, 2014).

La relevancia que tiene la web corporativa como principal canal de comunicación se hace patente con el crecimiento de la penetración de Internet y la generalización del uso de los nuevos dispositivos, tales como el smartphone o la tablet. De acuerdo con comScore, la audiencia digital española ya alcanza cerca de los 30 millones de usuarios en 2014. De dicha audiencia, más de 26 millones accede a través de un ordenador al medio digital y más de 23 millones lo hacen a través de su móvil (2). A este respecto, la accesibilidad, usabilidad y, especialmente, el responsive design de una web (Marcotte, 2011) son aspectos sobre los que se ha ido trabajando profusamente en los últimos años, cuya razón de ser es la comunicación con el usuario (3).

El término responsive design fue acuñado por Ethan Marcotte en 2010 aunque el consorcio internacional W3C ya incorporaba recomendaciones específicas para la web en móvil en el año 2008 (González; Marcos, 2013). Durante algunos años Google ha estado recomendado la optimización de las webs para móviles. Desde 2015, este buscador decidió penalizar a aquellas páginas que no estén adaptadas (Google, 2015).

\subsection{La gestión de Big Data como oportunidad para las agencias de medios}

En Internet aguardan inmensas y diferentes cantidades de datos que se multiplican rápidamente. Se trata del fenómeno big data cuyas características se resumen en las 3Vs de Russom (2011): volumen, variedad y velocidad.

Si se observan las funciones que las agencias de medios desarrollaban antes del advenimiento del mundo 
digital se comprende que la gestión de big data es una oportunidad que, entre muchas otras posibles, ofrece soluciones de comunicación también a la innovación de otras empresas. La correcta gestión de tales datos puede proporcionar ventajas competitivas a las organizaciones, según el 68\% de las 1144 estudiadas por IBM (2012). Potenciar este servicio podría, igualmente, implicar la necesidad de desarrollar software especializado y estimular, así, las innovaciones de mayor carácter tecnológico.

Los nuevos medios han introducido nuevas formas de comunicación, también, publicitaria, y en materia de investigación y planificación de medios publicitarios los retos se sitúan, sobre todo, en la medición y en la estrategia (Papí-Gálvez, 2014a).

En cuanto a la primera, la medición, y en relación a la audiencia online, hay que tener en cuenta que ésta ha constituído una condición necesaria para la planificación de medios. Esta disciplinaha evolucionado a medida que han ido emergiendo nuevas fuentes y metodologías (Lamas, 2010). Entre las ventajas de la medición electrónica actual destaca justamente la gran cantidad de datos que se puede almacenar en tiempo real para conocer el comportamiento de los usuarios ante los medios. Entre sus inconvenientes se puede señalar que no siempre sea posible controlar la vinculación entre máquina y persona con dicha medición (electrónica, censal o basada en el site) lo que genera serias limitaciones para poder calcular la cobertura (cantidad de personas expuestas a un contenido) y la frecuencia (cantidad de veces). También tiene limitaciones para proporcionar datos de orden más cualitativo tales como: influencia, percepción, relevancia, credibilidad o engagement. La aplicación de la tecnología big data podría ayudar a dar respuestas precisas (Papí-Gálvez, 2014b). Su gestión se ubicaría, así, en una etapa previa a la planificación de cualquier estrategia, cuya labor sería detectar los datos que son relevantes y transformarlos en conocimiento para tomar las decisiones correctas (de la Peña; Valle, 2014).

En cuanto a las estrategias, no cabe duda que los hábitos de consumo de medios de la población están cambiando. Las nuevas tecnologías flexibilizan los procesos y facilitan la comunicación líquida (Solana, 2010). Actualmente, además, los medios sociales, a través de los cuales cualquier usuario puede generar contenidos y soportes, forman parte de la rutina de cada vez un mayor número de personas. Las audiencias están fragmentadas aunque, como Internet llega a públicos muy homogéneos en alguna característica, también de naturaleza psicográfica, este medio puede contribuir a generar engagement (Papí-Gálvez, 2014c).

La agencia debe ser capaz de proponer estrategias con más información útil, en menos tiempo (Papí-Gálvez, 2014b), y proporcionar de este modo los contenidos adecuados que aporten valor a la marca insertándolos en el lugar y en el momento preciso para el usuario. La aplicación de big data en las agencias de medios favorece, así, la construcción de ese lugar de encuentro entre marca, audiencias y canales de una forma "más sensorial y eficaz" (Blanch, 2014, p. 204).

\subsection{Objetivos}

De estudios previos centrados en las primeras diez agencias de medios en España, según facturación, se desprende que estas empresas cumplirían con los primeros estadios del proceso comunicativo de la innovación, al poder extraer resultados sobre sus aspectos clave atendiendo, entre otras fuentes, a la información de sus webs corporativas (Papí-Gálvez, 2015, 2014b). Se trata de empresas en su gran mayoría multinacionales. Sin embargo, quedan pendientes preguntas sobre aquellas innovaciones que pudieran considerarse más próximas a la concepción tecnológica y, también, acerca de otras agencias no necesariamente internacionales. Igualmente, dada la popularización de los móviles de última generación, se debe reflexionar sobre la calidad de la comunicación de las webs, especialmente en lo que concierne al responsive design.

En consecuencia, este estudio pretende averiguar si las agencias de medios utilizan sus webs corporativas para comunicar innovaciones de carácter tecnológico cuidando el responsive design.

En concreto pretende:

1. Explorar el uso que hacen de las webs corporativas para comunicar servicios relacionados con la tecnología big data, 
2. Evaluar el responsive design de las webs de las principales agencias,

3. Detectar las debilidades, amenazas, fortalezas y oportunidades de su aplicación.

\section{MÉTODO}

Se concibió un estudio exploratorio basado en la investigación documental, como tipo de investigación encuadrada en el paradigma cualitativo, para observar las 20 primeras agencias, según el ranking de inversión gestionada de InfoAdex en 2013 (tabla 1). Se debe advertir que la segunda y tercera agencia del ranking forman actualmente el grupo Zenithoptimedia. Igualmente, Arena Media es parte de Havas Media Group.

\begin{tabular}{|l|l|}
\hline Ranking & $\begin{array}{l}\text { Inversión controlada por InfoAdex, } \\
\text { gestionada en 2013 (euros) }\end{array}$ \\
\hline HAVAS MEDIA & 562.363 .692 \\
\hline OPTIMEDIA & 278.429 .164 \\
\hline ZENITH & 235.738 .793 \\
\hline OMD & 228.814 .107 \\
\hline MEDIACOM IBERICA & 215.310 .467 \\
\hline ARENA MEDIA COMMUNICATIONS & 202.245 .552 \\
\hline YMEDIA & 159.664 .732 \\
\hline STARCOM & 153.610 .027 \\
\hline MAXUS & 82.246 .178 \\
\hline EQUMEDIA & 77.635 .575 \\
\hline UNIVERSAL MCCANN & 63.704 .393 \\
\hline VERITAS & 54.030 .476 \\
\hline MEC (FOCUS MEDIA) & $93.859 .896(40.954 .187)$ \\
\hline INITIATIVE & 44.025 .918 \\
\hline PHD & 22.790 .611 \\
\hline SERENDIPIA & 22.262 .862 \\
\hline DATAPLANNING & 18.031 .928 \\
\hline ALMA MEDIA & 10.275 .999 \\
\hline EMV.DOS & 8.797 .390 \\
\hline MEDIASAPIENS & 4.277 .897 \\
\hline TabIa. Rank Ageng & \\
\hline
\end{tabular}

Tabla 1. Ranking Agencias de Medios en España de InfoAdex.

Fuente: elaboración propia con datos de InfoAdex.

Para dar respuesta a los objetivos, el estudio se dividió en dos etapas:

En primer lugar se puso en marcha la fase analítica de observación tanto de la aplicación de big data en las agencias de medios como del responsive design de las webs consultadas.

La presencia de big data en el medio propio ser abordó a través del análisis textual de las webs. Para ello, se diseñó una plantilla en la que se recogía tres tipos de información:

1. si la web empleaba expresamente el concepto big data,

2. si aparecían otros conceptos cercanos a big data de acuerdo con la exposición que la empresa daba de sus funciones, del servicio o la denominación de dicho servicio (data in real time, data science...), y 
3. toda explicación del trabajo que realizaban las agencias, según sus webs, siempre que hubiera relación con big data, aunque no se utilizaran términos próximos (tabla 2).

Este análisis fue realizado por dos investigadores de forma independiente para poder compararlas con posterioridad, en virtud de la confiabilidad del método. Se controlaron, así, las posibles ambigüedades que pudieran desprenderse del tratamiento de los datos textuales. Esta información fue recogida y completada durante el segundo semestre de 2014.

\begin{tabular}{|c|c|}
\hline AGENCIA (NOMBRE): & WEB CONSULTADA: \\
\hline \multicolumn{2}{|l|}{ BIG DATA } \\
\hline La web, ¿utiliza este concepto? & 2. $\mathrm{NO}$ \\
\hline \multicolumn{2}{|l|}{$\begin{array}{l}\text { Breve descripción del servicio en el que } \\
\text { aparece (LITERAL): }\end{array}$} \\
\hline $\begin{array}{l}\text { OTROS CONCEPTOS RELACIONADOS } \\
\text { CON BIG DATA (FILA POR CONCEPTO) }\end{array}$ & $\begin{array}{l}\text { BREVE DESCRIPCION POR } \\
\text { CONCEPTO (LITERAL) }\end{array}$ \\
\hline $\begin{array}{l}\text { EXPLICACION DEL TRABAJO, } \\
\text { FUNCIONES O SERVICIOS QUE } \\
\text { APARECEN EN LA WEB RELACIONADOS } \\
\text { CON BIG DATA (FILA POR ACCION*) }\end{array}$ & BREVE DESCRIPCION (LITERAL) \\
\hline OBSERVACIONES: & \\
\hline
\end{tabular}

*Posibles ejemplos de denominaciones utilizadas para comunicar las funciones:

\begin{tabular}{|l|l|l|}
\hline ERC_Consultoría & Digital & Analítica Web \\
\hline Consultoría digital & Analytics/Reporting & Real Time Bidding \\
\hline Investigación & Métrica social & Puntos de contacto... \\
\hline
\end{tabular}

Tabla 2. Plantilla de recogida de información por agencia.

Fuente: elaboración propia.

En segundo lugar, se evaluó el responsive design haciendo uso de la prueba de optimización para móviles de Google que puede localizarse en la siguiente dirección: https://www.google.com/webmasters/tools/mobilefriendly.

Esta herramienta funciona introduciendo la url de la web y de forma automática Google escanea la página ofreciendo el resultado de la misma. Fueron analizadas las siguientes urls de las agencias (tabla 3). 
La segunda etapa del estudio consistía en una fase de diagnóstico que, basada en fuentes documentales y en los resultados de la primera parte, ayudaba a encontrar explicaciones. Esta etapa aplicaba el enfoque del análisis DAFO (Debilidades, Amenazas, Fortalezas y Oportunidades) para ordenar la información.

\section{RESULTADOS}

\subsection{Servicios Big Data en las Webs Corporativas}

Las agencias de medios con mayor gestión de la inversión publicitaria en España son internacionales por lo que se utilizaron webs corporativas de la empresa matriz traducidas o adaptadas al mercado español. Algunas, no obstante, son españolas, tales como Serendipia (SerendipSmarthinking), Dataplanning, Alma Media, EMV.DOS o Mediasapiens, a la cola del ranking. Las webs de estas empresas no proporcionaban suficiente información para poder hacer un análisis relacionado con los servicios ofrecidos que aplican, en concreto, tecnología big data. Por tanto, los resultados representan en mayor medida a las empresas con mayor inversión gestionada (las líderes).

El concepto big data aparece en las webs de las dos primeras (Havas Media y Zenithoptimedia), asociado a una metodología de carácter tecnológica utilizada para mejorar los servicios de investigación que le son propios. De la explicación de las funciones o servicios de otras agencias, con todo, se puede deducir la aplicación de metodologías de trabajo "inteligentes", además de otras consideraciones (atención a la creatividad y a los contenidos) tratadas en una investigación previa que pretendía observar el potencial innovador de las agencias de medios (PapíGálvez, 2015). El presente estudio se centra, en concreto, en big data.

Así, y de acuerdo con la web de Havas Media, la empresa se encarga de explorar nichos de oportunidad tanto para sus clientes actuales como para los futuros. Algunas de estas tendencias de medios en las que investiga son: las multipantallas, el social shopping, la localización, creación de contenido, realidad aumentada, big data y análisis predictivo, búsqueda inteligente y el life tracking. En su sección Data and Analytics explica:

"The digital marketplace generates huge amounts of timely, accurate and attributable data. The ability to capture, manipulate and understand this data is vital to craft, target and refine campaigns that build brands and generate sales. With a blend of sophisticated proprietary technology and really smart people $(\ldots)$ ".

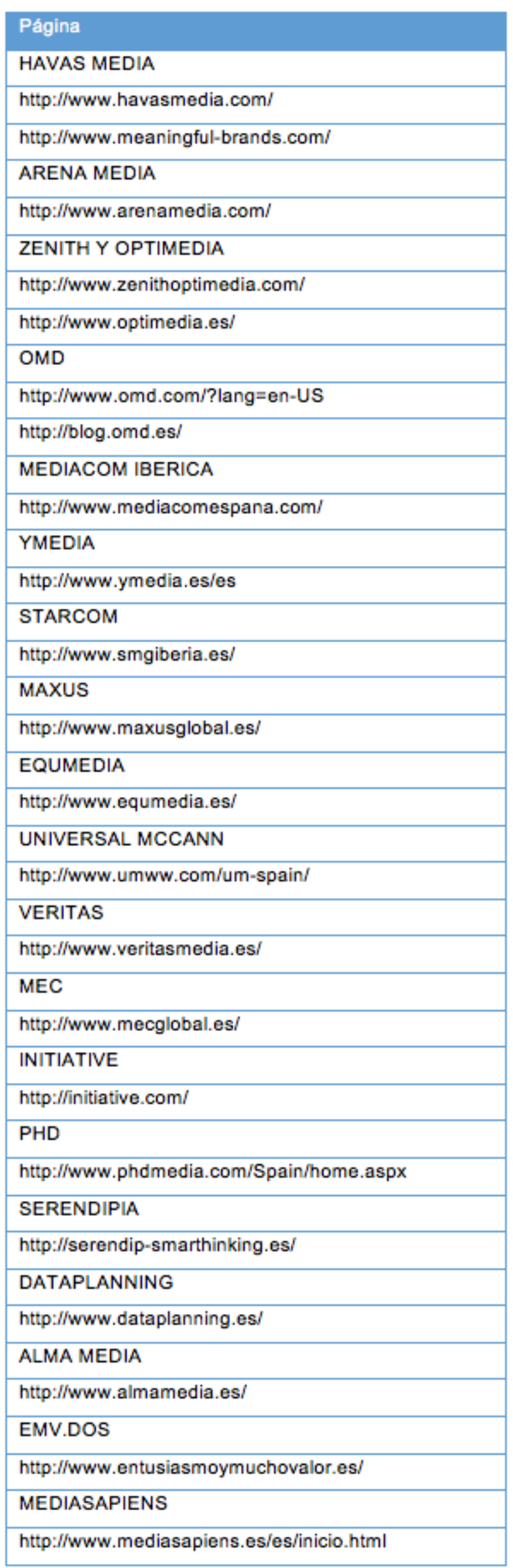

Tabla 3: Urls de las agencias.

Fuente: elaboración propia. 
también como fuente de información, en la línea de otros blogs de su propiedad. Define el servicio de big data como: “(...) la transformación de los datos en información, mediante su almacenamiento, análisis y visualización a través de dashboards en tiempo real".

En ambos casos han desarrollado tecnologías-herramientas específicas (por ejemplo, Artemis ${ }^{\circledR}$ de Havas Media) vinculada a estos servicios, y que son iniciativas que les hace poder ser consideradas empresas de innovación, también tecnológica.

Ninah es la consultora líder en analítica avanzada encargada de esta sección ( big data) que se introduce en 2014 en España de la mano del grupo Zenithoptimedia, tras llevar trabajando desde la década de los noventa en Reino Unido y haberse expandido a otros países europeos.

En consecuencia, las dos empresas líderes invierten en servicios de análisis basados en la minería de datos, aunque destaca especialmente el uso del término big data en el grupo Zenithoptimedia.

Entre las webs restantes, algunas agencias apuestan por el uso de otros términos que pudieran implicar el análisis de grandes cantidades de datos para transformarlos en información relevante (global data, business intelligence, business science, data science) o que se basan en tales análisis ( consumer insights, insights \& intelligence, planificación estratégica).

Algunas de las webs son muy genéricas en la exposición de sus funciones y servicios para averiguar si, en concreto, la gestión de datos masivos está presente o es ciertamente relevante en los mismos.

\subsection{Análisis de la optimización para móviles}

En la tabla 4 se observa que casi todas las webs consultadas están optimizadas para móviles. Se detectan sólo 6 páginas de las 22 analizadas con los siguientes problemas:

1. El texto es demasiado pequeño para leerlo.

2. Los enlaces están demasiado juntos.

3. No se ha definido la ventana gráfica para dispositivos móviles.

a) El contenido es más ancho que la pantalla.

b) Utiliza plug-ins incompatibles. 


\begin{tabular}{|c|c|c|}
\hline Página & Optimizada & No Optimizada \\
\hline \multicolumn{3}{|l|}{ HAVAS MEDIA } \\
\hline http://www.havasmedia.com/ & $\mathrm{x}$ & \\
\hline http://www.meaningful-brands.com/ & $\mathrm{x}$ & \\
\hline \multicolumn{3}{|l|}{ ARENA MEDIA } \\
\hline http://www.arenamedia.com/ & $\mathrm{x}$ & \\
\hline \multicolumn{3}{|l|}{ ZENITH Y OPTIMEDIA } \\
\hline http://www.zenithoptimedia.com/ & $\mathrm{X}$ & \\
\hline http://www.optimedia.es/ & $\mathrm{x}$ & \\
\hline \multicolumn{3}{|l|}{ OMD } \\
\hline http://www.omd.com/?lang=en-US & $\mathrm{x}$ & \\
\hline http://blog.omd.es/ & $\mathrm{x}$ & \\
\hline \multicolumn{3}{|l|}{ MEDIACOM IBERICA } \\
\hline http://www.mediacomespana.com/ & & $1,2,3,4$ \\
\hline http://www.ymedia.es/es & & $1,3,4$ \\
\hline \multicolumn{3}{|l|}{ STARCOM } \\
\hline http://www.smgiberia.es/ & & $2,3,4$ \\
\hline \multicolumn{3}{|l|}{ MAXUS } \\
\hline http://www.maxusglobal.es/ & $\mathrm{x}$ & \\
\hline \multicolumn{3}{|l|}{ EQUMEDIA } \\
\hline http://www.equmedia.es/ & $\mathrm{x}$ & \\
\hline \multicolumn{3}{|l|}{ UNIVERSAL MCCANN } \\
\hline http://www.umww.com/um-spain/ & $\mathrm{x}$ & \\
\hline \multicolumn{3}{|l|}{ VERITAS } \\
\hline http://www.veritasmedia.es/ & & $1,2,3,4$ \\
\hline \multicolumn{3}{|l|}{ MEC } \\
\hline http://www.mecglobal.es/ & $\mathrm{x}$ & \\
\hline \multicolumn{3}{|l|}{ INITIATIVE } \\
\hline \multicolumn{3}{|l|}{ http://initiative.com/ } \\
\hline \multicolumn{3}{|l|}{ PHD } \\
\hline http://www.phdmedia.com/Spain/home.aspx & & $1,2,3,4$ \\
\hline \multicolumn{3}{|l|}{ SERENDIPIA } \\
\hline http://serendip-smarthinking.es/ & $\mathrm{X}$ & \\
\hline \multicolumn{3}{|l|}{ DATAPLANNING } \\
\hline http://www.dataplanning.es/ & & $2,4,5$ \\
\hline \multicolumn{3}{|l|}{ ALMA MEDIA } \\
\hline http://www.almamedia.es/ & $\mathrm{x}$ & \\
\hline \multicolumn{3}{|l|}{ EMV.DOS } \\
\hline http://www.entusiasmoymuchovalor.es/ & $\mathrm{x}$ & \\
\hline \multicolumn{3}{|l|}{ MEDIASAPIENS } \\
\hline http://www.mediasapiens.es/es/inicio.html & & $1,2,3$ \\
\hline
\end{tabular}

Tabla 4: Resultados de la prueba de optimización para móviles de Google.

Fuente: https://www.google.com/webmasters/tools/mobile-friendly

\subsection{Entorno: Amenazas y Oportunidades}


En el entorno que rodea a las agencias de medios, existen una serie de factores que podrían limitar la predisposición de éstas hacia la inmersión en la gestión de big data. Uno de los más importantes comprende la situación económica por la que pasa actualmente España. La crisis económica supondría la principal amenaza para la aplicación ya que reduce los ánimos de emprendimiento y dificulta la inversión en nuevos negocios (CEOE, 2014).

Otras posibles amenazas son las mentalidades más conservadoras partidarias de sistemas de medición tradicionales por falta de conocimiento, pues la gestión de medios online establece ahora sus bases en torno al concepto de insight aplicado a la investigación y a la planificación. Y la estrategia de medios ha cambiado al POE(S) (medios pagados, paid, propios, owned, ganados, earned, e incluso compartidos, shared).

En España, el buscador más utilizado es Google (Papí-Gálvez, 2014a). Las decisiones que tome la empresa relacionadas con los parámetros que intervienen en el posicionamiento de las webs pueden ser consideradas, hoy en día, bien una amenaza bien una oportunidad. Desde 2015, se penaliza a aquellas páginas que no tengan responsive design, por lo que se trata de una amenaza para aquellos medios online que no se han adaptado a la realidad multidispositivo (Google, 2015).

Sin embargo, el auge de las tecnologías de la información y de la comunicación y, en concreto, de los medios online convierte la gestión de los datos masivos en una buena alternativa de negocio. La globalización de los medios y las extensas cantidades de información que existen en la red incentivan el desarrollo de aplicaciones estrechamente relacionadas con el mundo online. Esta situación generó incluso nuevas experiencias e innovadores negocios como las startup (Startup Genome y Telefónica Digital, 2012).

En España se está fomentando la innovación de las pequeñas y medianas empresas (Secretaría del Estado de Investigación, 2015). Se trata de medidas que pretenden responder al proyecto Europa 2020. Existen, así, oportunidades que podrían animar a las agencias medianas a integrar los datos masivos en sus productos y procesos, entre otro tipo de cambios producidos ante la sociedad digital (en las agencias líderes del ranking) que demuestran el potencial de innovación de tales empresas.

En este contexto, la comunicación de la innovación se hace necesaria. Los medios digitales y los dispositivos móviles elevan las oportunidades para dar a conocer los servicios ofrecidos ajustados a esta nueva realidad.

\subsection{Aplicación: Limitaciones y Fortalezas}

Entre sus limitaciones, big data tiene detractores ya que consiste en manejar amplias bases de datos con alto contenido personal. Su tratamiento podría llegar a invadir la privacidad de los usuarios. Por ello, es necesario no cruzar los límites de la legalidad y reforzar la fidelidad de los usuarios a través de buenas prácticas de comportamiento (Zenithoptimedia, 2015).

Igualmente, la aplicación de la tecnología big data (y de la estrategia basada en la misma) precisa equipos multidisciplinares y profesionales cualificados. Además, la gestión de datos masivos, y el tratamiento de varias databases, requiere una enorme infraestructura tecnológica (Abad, 2014).

En cuanto a las fortalezas, utilizar técnicas de big data en una agencia de medios podría mejorar sus competencias, como empresa (Davenport, 2014), en las tareas propias de la agencia, como la medición, la estrategia o la planificación (Papí-Gálvez, 2014a).

De hecho, las nuevas tendencias en investigación y planificación demandan otro tipo de información sobre el usuario. Los aspectos sociodemográficos ya no son suficientes para la segmentación del público objetivo de medios. La publicidad en Internet es cada vez más específica, por lo que la planificación también debe personalizarse según sea requerida por los perfiles.

Por tanto, gestionar los datos masivos facilita el diseño de estrategias específicas para usuarios muy homogéneos en algunas características que interesen. También sería posible tener modelos predictivos sobre las conductas de los grupos sociales, lo que confiere una ventaja significativa para conseguir mayor eficacia en los planes. 
Asimismo, implantar big data como estrategia de negocio podría impulsar un mejor desarrollo de la actividad empresarial y la obtención de mayores beneficios a largo plazo. El análisis de grandes volúmenes de datos permitiría comprender el completo funcionamiento de los sistemas que componen la agencia y trabajar de una forma más rápida y eficaz.

Si la innovación se lleva a cabo y se ofrecen nuevos servicios atendiendo a las fortalezas de la gestión de big data para la comunicación publicitaria, el medio propio debe ser un ejemplo justamente de la adopción de las nuevas tecnologías. La web puede hablar del nivel de competencia de la agencia, por lo que debe ser considerada como una fortaleza en sí misma si, además de otras cuestiones relacionadas con su diseño, cumple con los estándares de accesibilidad y tiene en cuenta la usabilidad y el responsive design.

\section{CONCLUSIONES}

El usuario es cada vez más importante en el proceso estratégico de la comunicación publicitaria, por ello, es necesario extraer un mayor volumen de información acerca del internauta, que no se base únicamente en características sociodemográficas, sino en datos psicológicos y modelos de conducta.

Las agencias buscan una planificación personalizada según usuarios. Necesitan, así, poder gestionar toda esta información relevante que encuentran sobre los mismos. Es aquí donde entra en escena la gestión de big data.

Si se aprovechan las oportunidades, la gestión de big data es el camino idóneo para optimizar la labor de las agencias y alcanzar un conocimiento más amplio del entorno que les rodea y del público. Todo ello les permitiría enfocar sus estrategias con mayores garantías de éxito y generar beneficios a medio y largo plazo.

Tales oportunidades y fortalezas se ven reflejadas en la aplicación de esta tecnología en las grandes agencias de medios, pioneras en innovaciones que les proporcionan ventajas competitivas o que forman parte de un grupo que las tiene. En cambio, las agencias que están a la cola del ranking, pese a su elevada gestión de la inversión publicitaria, parecen representar los inconvenientes (amenazas y limitaciones) que se han ido exponiendo. La cuestión para tales agencias es si merece la pena asumir los costes que conlleva la implantación de un sistema de gestión de big data, más allá de los métodos habituales de investigación, en un mercado como el actual.

No obstante, si se atiende al comportamiento de las dos agencias líderes en España (Havas Media y Zenithoptimedia), que refleja lo que está ocurriendo a nivel internacional, parece que la aplicación de la gestión de los datos masivos como parte del proceso de trabajo de las agencias de medios será inevitable. Como se ha expuesto en estudios previos, y a juzgar por la comunicación que de sus servicios hacen en las webs, conocen la importancia de transmitir conceptos tales como inteligencia, tecnología, investigación, análisis o innovación.

Con todo, esta investigación también detecta que la comunicación online, a través de las páginas corporativas, podría y tendría que ser mejorada en algunas de ellas tanto en contenido como en aspectos técnicos. Este estudio ha evaluado las webs según su responsive design y, a la luz de los resultados, concluye que pese a que la mayor parte de ellas están optimizadas, al tratarse de las agencias que más facturan en España y dedicándose, como se dedican, a la comunicación, se puede considerar 6 webs de 22 un número importante de pagina a revisar. . En consecuencia, se debe analizar con mayor profundidad las webs atendiendo no sólo al contenido sino también a otros elementos que afectan al diseño y a su comunicación. Por ello, se propone autoevaluar las webs teniendo en cuenta tanto el responsive design como la usabilidad y accesibilidad.

En definitiva, la transformación del escenario mediático afecta a la actividad de las agencias de medios. Por ello, las agencias españolas deberán encontrar fórmulas para poder incorporar el análisis de los datos masivos en sus servicios si aspiran a diseñar estrategias y planificaciones más eficaces en la sociedad digital pero, además, también tienen que hacer un mayor esfuerzo para comunicar tales innovaciones a través de sus webs. 
ABAD, S. (2014): Big data en números. Informes OBS (Online Business School), [en línea]. [Consulta 20 de junio]. <http://goo.gl/aZeGu3>

BLANCH, A. (2014): "Hacia una planificación en tiempo real". adComunica: Revista Científica de Estrategias, Tendencias e Innovación en Comunicació, nº 7, pp. 203-204.

BOE (2014): Ley 27/2014, de 27 de noviembre, del Impuesto sobre Sociedades. [en línea]. [Consulta 20 de junio]. <http://goo.gl/8ugJBa>

CEOE (2014): El estado de la innovación empresarial en España en 2014. Madrid: CEOE.

Comisión Europea (2010): Iniciativa emblemática de Europa 2020 Unión por la innovación [en línea]. [Consulta 15 de junio]. <http://goo.gl/bBi93u>

- (2014): Research and innovation as sources of renewed growth [en línea]. [Consulta 15 de junio]. $<$ http://goo.gl/T3rch8>

- (s.f.): Europe 2020 [en línea]. [Consulta 15 de junio]. <http://ec.europa.eu/europe2020/index_es.htm> COTEC (2004): Comunicar la innovación. Madrid: Fundación Cotec para la Innovación Tecnológica.

COTEC (2015): Informe Cotec. Tecnología e Innovación. Madrid: Fundación Cotec para la Innovación Tecnológica.

CORPORATE EXCELLENCE (2015): "Cómo comunicar la innovación para crear un gran valor de marca y construir una excelente reputación”. Insights \& Trends, Documentos de estrategia, n 150, pp. 1-4.

DAVENPORT, Th. (2014): Big Data at Work: Dispelling the Myths, Uncovering the Opportunities. USA: Harvard Business School.

De la Peña, C.; Valle, A. (2014): "Del dato a la toma de decisiones, pasando por el conocimiento", HarvardDeusto. Márketing y Ventas, $\mathrm{n}^{\circ} 127$, pp. 34-41.

GOOGLE (2015): Finding more mobile-friendly search results. Web Master Central Blog, [en línea]. [Consulta 25 de septiembre]. <http://googlewebmastercentral.blogspot.com.es/2015/02/finding-more-mobile-friendlysearch.html>

GONZÁLEZ, D.; MARCOS, M. C. (2013). Responsive web design: diseño multidispositivo para mejorar la experiencia de usuario. BiD: textos universitaris de biblioteconomia i documentació. desembre, $\mathrm{n}^{\circ} 31$, [en línea]. [Consulta 18 de junio]. <http://bid.ub.edu/pdf/31/es/gonzalez2.pdf>

IBM (2012): Analytics: el uso de big data en el mundo real. Cómo las empresas más innovadoras extraen valor de datos inciertos. Madrid: IBM España.

INFOADEX (2014): Inversión publicitaria gestionada en 2013 por agencias de medios. Madrid: InfoAdex.

LAMAS, C. (2010): "Los Medios Interactivos y su Publicidad. La medición de audiencias", Revista Telos, n 82, pp. 1-8.

McGRATH, R. G. (2013): "The end of competitive advantage: How to keep your strategy moving as fast as your business". Harvard Business Review Press.

NIELSEN, J. (2000): Usabilidad: Diseño de sitios web. Madrid:

Prentice Hall.

PAPÍ-GÁLVEZ, N. (2014a): "Los medios online y la ¿crisis? de la planificación de medios publicitarios", adComunica. Revista Científica de Estrategias, Tendencias e Innovación en Comunicación, nº 7, pp. 29-48. 
- (2014b): Retos de las agencias de medios ante el mundo online. Claves para los estudios de publicidad. En MENDIETA, A.; SANTOS, C.J. Líneas emergentes en la investigación de vanguardia. Madrid: McGrawHill Education, pp. 475-485.

- (coord.) (2014c): Claves de la publicidad online: Fundamentos, Herramientas y Retos. Madrid: AIMC.

- (2015): "Los nuevos medios y las empresas innovadoras. El caso de las agencias de medios", El profesional de la información, vol. 24 (mayo-junio), nº 3, pp. 301-309.

PÉREZ-LATRE, F.; SÁNCHEZ TABERNERO, A.: Fundamentals for the Transformation of Media Companies in the Digital Age. En HERRERO, M.; WILDMAN, S. (2015): The Business of Media: Change and Challenges.

Media XXI/Porto: Formalpress, pp. 53-62

RUSSON, Ph. (2011): Big data analytics. TDWI Best practices report. TDWI Research, IBM.

Secretaría de Estado de Investigación (2015): Desarrollo e Innovación, Actuaciones de fomento de la innovación. Ministerio de Economía y Competitividad, [en línea]. [Consulta 20 de junio]. <http://goo.gl/IAtihH>

SOLANA, D. (2012): Postpublicidad. Reflexiones sobre una nueva cultura publicitaria en la era digital. Barcelona: Doubleyou.

STARTUP GENOME; TELEFÓNICA DIGITAL (2012): Startup Ecosystem Report 2012. Startup Genome y Telefónica Digital.

VANHAVERBEKE, W.; CHESBROUGH, H.: A classification of Open Innovation and Open Business Model. En Chesbrough, H.; Vanhaverbeke,W.; West, J. (2014): New Frontiers in Open Innovation. Oxford University Press. pp. 50-70.

ZENITHOPTIMEDIA (2015): El Big Data según los expertos: implantación, retos y situación española. BlogginZenith [en línea]. [Consulta 20 de junio]. <http://goo.gl/Mnr4Kg>

(1) A este respecto, la Ley $27 / 2014$, de 27 de noviembre, del Impuesto sobre Sociedades dirige las actividades de investigación y de desarrollo a la "fabricación de nuevos materiales o productos o al diseño de nuevos procesos o sistemas de producción" y excluye los estudios de mercados y las prospecciones de ciencias sociales como acciones I+D+i: "Se considerará innovación tecnológica la actividad cuyo resultado sea un avance tecnológico en la obtención de nuevos productos o procesos de producción o mejoras sustanciales de los ya existentes" (BOE, 2014: 96998).

(2) Hay que advertir que las poblaciones no son iguales. Sólo los usuarios a través de ordenador incluyen niños por lo que el móvil se presenta ya como el principal dispositivo de acceso a Internet.

(3) Los estándares para la accesibilidad están establecidos (WCAG). La usabilidad implica varias etapas en la creación de, por ejemplo, una aplicación informática, pero siempre se define otorgándole un peso mayor a la experiencia del usuario con la tecnología. De hecho, una de las primeras definiciones de usabilidad, pronunciada por Nielsen, incluía aspectos tales como la facilidad para aprender su funcionamiento por parte del usuario, eficiencia o rapidez de manejo, capacidad de recuerdo, reducción de los errores en el uso de la aplicación y la satisfacción subjetiva. En cuanto al responsive web design se trata de adaptar de forma adecuada la web para que esta sea accesible y fácilmente navegable desde cualquier dispositivo.

\section{BREVE SEMBLANZA DE LOS AUTORES}

Natalia Papí Gálvez es profesora titular de Publicidad y Relaciones Públicas en la Universidad de Alicante, 
licenciada en Sociología con la especialidad en Marketing y Publicidad y doctora por la Universidad de Alicante. Dirige el "Grupo de estudios de comunicación estratégica (E-COM)" que nace con una doble vocación: investigar sobre temas actuales que conciernen a la comunicación y orientar a futuros investigadores.

Raquel García Bonal es Graduada en Publicidad y Relaciones Públicas por la Universidad de Alicante. Completa su formación en la Escuela Superior de Publicidad de Madrid y realiza cursos sobre publicidad digital avalados por la IAB. Ha colaborado con agencias online tales como Human Level Communications y Synergy Internet.

Ámbitos. Revista Internacional de Comunicación, n.31, edición de invierno, 2016.

Recibido: 26/03/2015

Aprobado: 25/04/2015 